\title{
Magnetic reconnection during eruptive magnetic flux ropes ${ }^{\star}$
}

\author{
Z. X. Mei ${ }^{1,2}$, R. Keppens ${ }^{1}$, I. I. Roussev ${ }^{3,1}$, and J. Lin ${ }^{2,4}$ \\ ${ }^{1}$ Centre for mathematical Plasma Astrophysics, Department of Mathematics, KU Leuven, Celestijnenlaan 200B, 3001 Leuven, \\ Belgium \\ e-mail: meizhixing@ynao.ac.cn \\ 2 Yunnan Observatories, Chinese Academy of Sciences, Kunming, Yunnan, PR China \\ 3 Division of Atmospheric and Geospace Sciences, National Science Foundation, Arlington, Virginia, USA \\ ${ }^{4}$ Center for Astronomical Mega-Science, Chinese Academy of Sciences, Chaoyang District, Beijing, PR China
}

Received 10 May 2017 / Accepted 13 July 2017

\begin{abstract}
Aims. We perform a three-dimensional (3D) high resolution numerical simulation in isothermal magnetohydrodynamics to study the magnetic reconnection process in a current sheet (CS) formed during an eruption of a twisted magnetic flux rope (MFR). Because the twist distribution violates the Kruskal-Shafranov condition, the kink instability occurs, and the MFR is distorted. The centre part of the MFR loses its equilibrium and erupts upward, which leads to the formation of a 3D CS underneath it.

Methods. In order to study the magnetic reconnection inside the CS in detail, mesh refinement has been used to reduce the numerical diffusion and we estimate a Lundquist number $S=10^{4}$ in the vicinity of the CS.

Results. The refined mesh allows us to resolve fine structures inside the 3D CS: a bifurcating sheet structure signaling the 3D generalization of Petschek slow shocks, some distorted-cylindrical substructures due to the tearing mode instabilities, and two turbulence regions near the upper and the lower tips of the CS. The topological characteristics of the MFR depend sensitively on the observer's viewing angle: it presents as a sigmoid structure, an outwardly expanding MFR with helical distortion, or a flare-CS-coronal mass ejection symbiosis as in 2D flux-rope models when observed from the top, the front, or the side.
\end{abstract}

Key words. magnetic reconnection - Sun: flares - Sun: coronal mass ejections (CMEs) - magnetohydrodynamics (MHD) instabilities

\section{Introduction}

Magnetic flux ropes (MFRs) are essential for many eruptive phenomena on the sun because they represent arch-shaped magnetic structures that contain significant non-potential magnetic energy. It is well known that the solar atmosphere is occupied by many coronal loops with distorted arch-shapes (Janvier et al. 2015). Their non-potential magnetic energy comes from the emergence of helical magnetic flux from the convection zone and/or they accumulate energy continuously by the slow driving motions of photosphere plasma. When the non-potential energy of the MFRs reaches the critical value, the overall equilibrium of magnetic structure can be lost dynamically (Forbes \& Priest 1995; Lin \& Forbes 2000; Kliem et al. 2014), the increasing magnetic twist inside a MFR may trigger kink instabilities (Baty 2000; Török et al. 2004; Mei et al. 2017), or the overarching magnetic configuration may not be able to constrain the MFR expansion (Kliem \& Török 2006).

The dynamical evolution of MFRs lead to rapid changes in magnetic configuration and the appearance of net electric current concentrated in localized regions. Underneath an erupting MFR current sheets (CSs) appear, which represent locations of energy release by magnetic reconnection (Mei et al. 2012; Kliem et al. 2013; Nishida et al. 2013; Archontis et al. 2014). Observations of magnetic reconnection during eruptive events have been reported by many works. Many authors have deduced the magnetic reconnection sites during eruptions from the presence of the hard X-ray sources above the flare loop tops

\footnotetext{
$\star$ The movie associated to Fig. 2 is available at http://www . aanda.org
}

(Masuda et al. 1994; Su et al. 2013) or by the reconnecting inflow and outflow (Li \& Zhang 2009; Takasao et al. 2012). Some authors have also presented direct evidence of the long straight reconnecting CSs and plasma blobs moving along them by SOHO/LASCO C2 and UVCS (Lin et al. 2005; Schanche et al. 2016).

In recent decades, many theoretical works have studied the eruptive MFRs and the consequent magnetic reconnection in model atmosphere and magnetic topologies. Using analytical models, one can perform parametric studies of MFR physics (Forbes \& Priest 1995; Titov \& Démoulin 1999; Lin et al. 2002). This has been followed up by many numerical simulations involving the formation of MFRs (Leake et al. 2013; Archontis et al. 2014), the trigger mechanism of eruption, such as the loss of equilibrium and the kink instabilities of MFR (Baty et al. 1998; Roussev et al. 2003; Török et al. 2004; Mei et al. 2017), and the consequent magnetic reconnection (Nishida et al. 2009, 2013; Mei et al. 2012; Guidoni et al. 2016).

Here, we perform a high resolution 3D isothermal MHD simulation based on the analytic TD99 flux rope model (Titov \& Démoulin 1999). In contrast to the other works, we study the 3D morphology and the consequent reconnecting CS during the eruption of MFR triggered by the kink instability. Specifically, we adopt mesh refinement technology to resolve substructures inside the CS, self-consistently formed during the eruption. In Sect. 2 we describe the initial magnetic configuration, the boundary conditions, and the mesh refinement strategy. In Sect. 3 the results of the eruptive MFR and substructures inside the CS are presented. In Sect. 4 we summarize this work. 


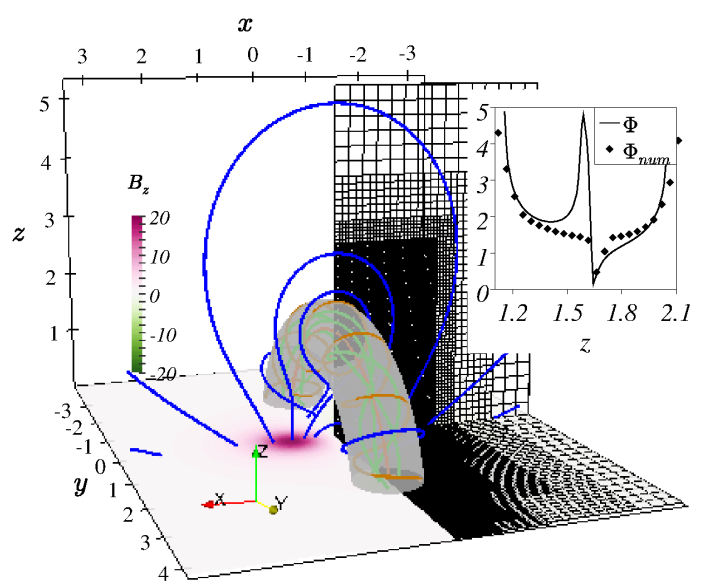

Fig. 1. Initial magnetic structure (coloured curves with green for internal magnetic flux rope (MFR) field lines, orange for the boundary MFR, and blue for the surrounding); the distribution of normal magnetic field (red-green shadings) on the bottom plane $z=0$ and a red line marks magnetic axis of MFR. The translucent iso-surface of current is for $j=0.4 j_{\max }$. Here, $j_{\max }$ is the maximum value of current inside initial MFR. The insert in the top right corner gives the twisted turns of magnetic field lines obtained by two methods: solid black line for $\Phi$ calculated by Eq. (1) and symbols for numerical turns $\Phi_{\text {num }}$ by integrating magnetic field around MFR magnetic axis.

\section{Numerical simulation set-up}

The initial magnetic structure is based on the TD99 model for solar eruptive events, which is constructed by superposing an MFR on a background field, where we allow for an internal pressure distribution (Titov \& Démoulin 1999; Mei et al. 2017). As shown in Fig. 1, a first part comes from a MFR with minor radius $a$, major radius $R$, and total toroidal current, $I$, uniformly distributed over its circular cross section. Another part is a background field due to a pair of magnetic sources $\pm q$ separated by a distance $L$, which are on the axis of symmetry of MFR and buried a distance $d$ under the photosphere $z=0$. The external equilibrium of MFR is realized by the confinement due to the background field from the magnetic sources. Inside the MFR, the gas pressure and the magnetic pressure of the toroidal field together achieve the internal equilibrium of the MFR. The relevant parameters for the TD99 model are set to $R=2.2$, $a=0.48, d=0.6, L=0.7, q=27.2$, and $I=2.89$ (similar to the case of inftwist in Mei et al. 2017). For convenience, all physical quantities are dimensionless with units $5 \times 10^{9} \mathrm{~cm}$, $1.28 \times 10^{7} \mathrm{~cm} \mathrm{~s}^{-1}, 3.89 \times 10^{2} \mathrm{~s}, 1.67 \times 10^{-14} \mathrm{~g} \mathrm{~cm}^{-3}, 2.76 \mathrm{~Pa}$, $5.89 \mathrm{G}$, and $2.95 \times 10^{11}$ A for length, velocity, time, density, pressure, magnetic field, and current, respectively.

A key parameter of the kink instability is the twisted turns $\Phi(r)$ for a straight MFR with length $2 R \cos ^{-1}(d / R)$ quantified by

$\Phi(r)=\frac{R B_{\mathrm{p}}(r)}{r B_{\mathrm{t}}(r)} \cos ^{-1}\left(\frac{d}{R}\right)$,

where $r$ is the distance away from the axis of the straight MFR, and we distinguish the strengths of the poloidal from the toroidal (along the MFR) fields. The twisted turns distribution of the MFR is shown in the inset of Fig. 1. The black solid line gives the twisted turns distribution of the MFR along the $z$-axis, which is based on the analytic results of TD99 and Eq. (1). Because the toroidal field vanishes outside the MFR, the twisted turns on the surface of MFR become infinite. Another more reliable value
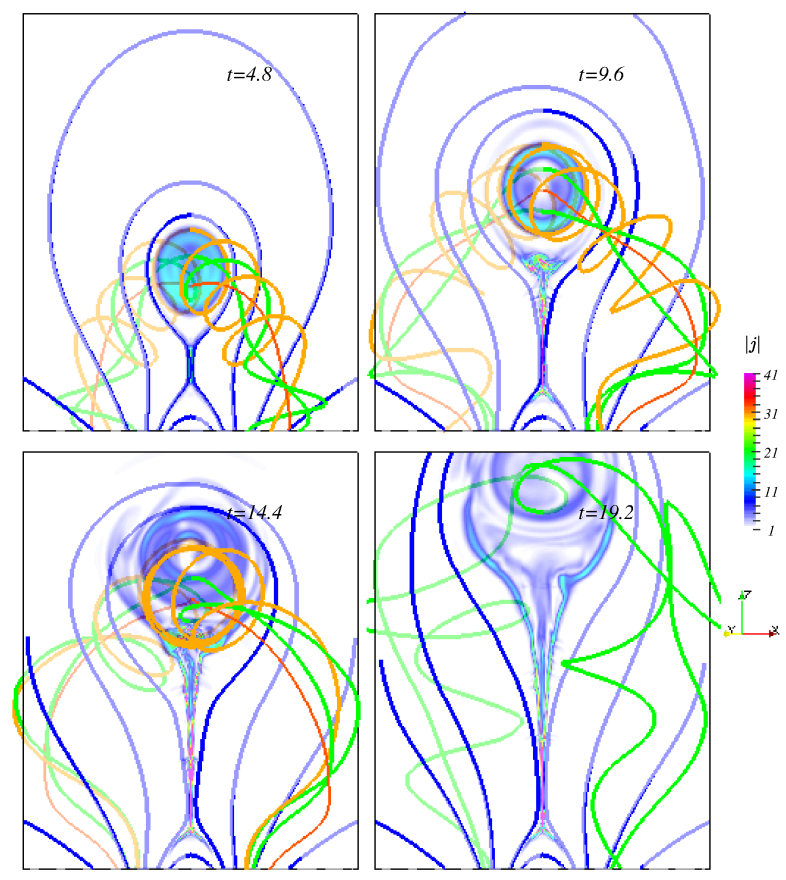

Fig. 2. Evolution snapshots of MFR at different times. Coloured curves are magnetic field lines outside and inside the MFR (blue and green) and on the surface of the MFR (orange). Translucent colours illustrate the current distribution on the cut $y=0$. An animation for MFR evolution is available online.

for the twisted turns is the numerical value $\Phi_{\text {num }}$, which considers the twisted turns that are resolved numerically. It is shown by the dotted line in Fig. 1, which is calculated by sampling points on the $z$-axis. These $\Phi_{\text {num }}$ values are calculated by the integration of the angular variation along the magnetic axis curve of MFR (Guo et al. 2013). Its value on the MFR surface is about five turns, which means the MFR is kink unstable.

The isothermal MHD equations in our numerical simulation are solved by the MPI-parallelized Adaptive Mesh Refinement code (MPI-AMRVAC, Keppens et al. 2012; Porth et al. 2014). We employ a third-order accurate shock-capturing finite volume spatial discretization, based on a Harten-Lax-van Leer approximate Riemann Solver, a third-order limiter in the reconstruction procedures (Čada \& Torrilhon 2009), and a three-step RungeKutta time marching. The computation domain is a 3D box of sizes $-4 \leq x \leq 4,-4 \leq y \leq 4$, and $0 \leq z \leq 12$ in Cartesian coordinates. A non-uniform mesh with four refinement levels is employed to follow the geometrical expansion of MFR. Around the CS formed during eruption, we adopt another two levels of refinement that use an automated error detection on the magnetic field. Thus, an effective resolution of $1728^{3}$ in the vicinity of the CS tracks the CS details. Here, the refinement ratios between grid levels are fixed to 2 . At the bottom of the simulation box, the line-tied condition is used to model the effect of the photospheric environment by fixing all three components of velocity at $z=0$ to zero, and keeping the magnetic field in the ghost cells unchanged. For the other boundaries, we adopt the open boundary condition that all the physical variables in the ghost cells should use an extrapolation of internal grid points.

\section{Numerical results}

Because of the large twist turns on the surface of MFR, the kink instability twists the MFR immediately after the launch 


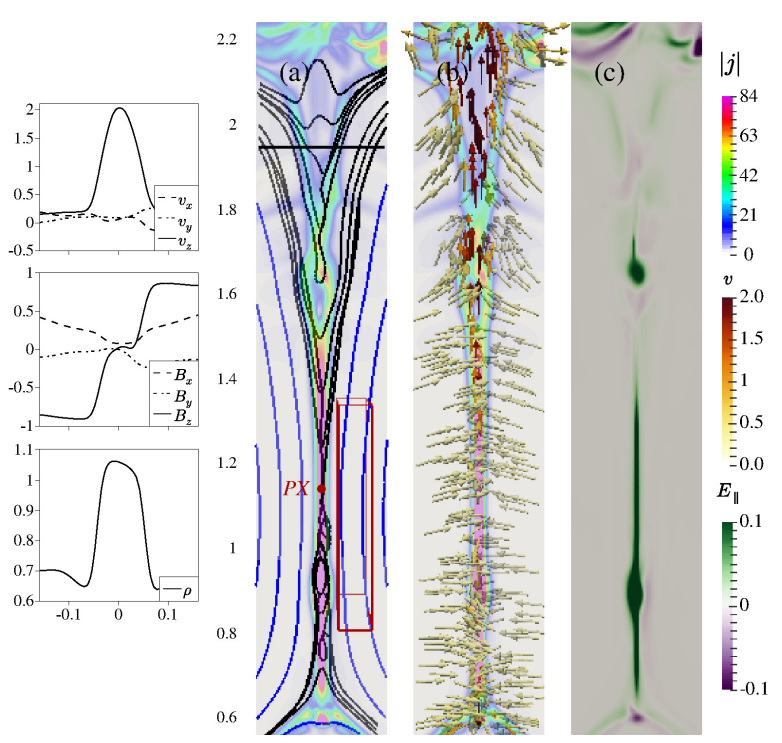

Fig. 3. Fine structures inside the current sheet (CS) at $t=9.6$. a) Current distribution on the cut $y=0$; magnetic field lines passing through points uniformly distributed on the $z$-axis (black lines) and outside the CS (blue lines). The inserts on the left side give the variation of physical quantities along the line marked on this panel. A red point $P X$ and a red box are marked for the evaluation of reconnection rates. $b$ ) Distributions of plasma velocity (arrows) in the vicinity of the CS. $c$ ) Distribution of parallel electric field $E_{\|}=\left(\eta_{\text {num }} \nabla \times \boldsymbol{B}\right) \cdot \hat{\boldsymbol{B}}$.

of simulation. The evolution snapshots are given in Fig. 2. The 3D magnetic structures at different times are shown by colour curves. The magnetic field in MFR is twisted continuously so that the central part of MFR loses its equilibrium. Similar to the 2D flux-rope models (Lin \& Forbes 2000; Mei et al. 2012), the MFR erupts upward and a 3D CS forms under the MFR, as shown by the current distribution (translucent colours) on the cut at $y=0$. During the eruption, the magnetic field lines around the MFR are stretched, while the magnetic fields under the MFR converge toward one another. Thus, a 3D CS forms under the MFR and continuously grows in length along the $z$-direction.

The growing CS shows a bifurcating structure, which includes two pairs of Petschek slow shocks separating the reconnection outflow from the reconnection inflow. Figure 3 gives more details of this bifurcating structure at $t=9.6$. In panel a, the magnetic field lines passing through discrete points on the $z$-axis (black) and near the CS (blue) are given. Three insets on the left side of the figure give the distributions of plasma velocities, magnetic fields, and density along the line marked on this panel a. It is evident that these physical parameters undergo stepwise changes, and this discontinuous structure had been identified as the slow mode shock in several 2D theories and numerical results (Vasyliunas 1975; Forbes \& Malherbe 1986; Mei et al. 2012). The reconnection rates in the CS are estimated in two ways: by evaluating it near the primary $\mathrm{X}$-point $P X$ and by averaging quantities over a red box marked in panel a. Here the reconnection rate is quantified as the ratio between reconnection inflow $\left|v_{x}\right|$ and the local Alfvén speed, $v_{A}$. They are equal to 0.2 at $\mathrm{PX}$ and 0.085 in the box, respectively. These values indicate that the ongoing reconnection is much faster than the Sweet-Parker reconnection rate.

In panel $b$, the distribution of the plasma velocity is given. The reconnection inflow approaches the CS from both sides, and is accelerated to the value of Alfvén speed in the upstream when it crosses the slow shock into the interior of CS. Therefore, the

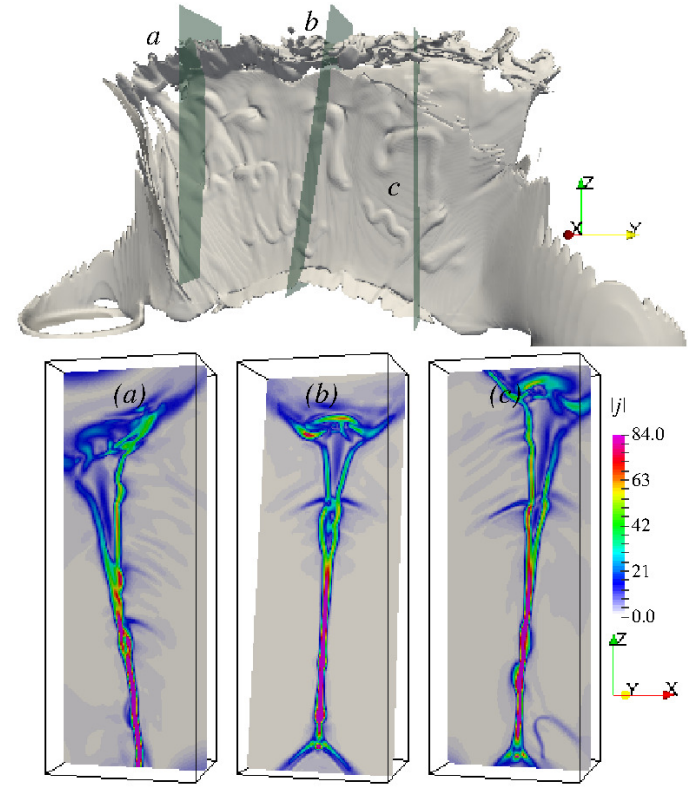

Fig. 4. Snapshot of the current sheet at $t=9.6$. Upper panel: current isosurface with value $j=24$, where $3 \mathrm{D}$ distorted-cylindrical substructures can be seen. Lower panels: current distributions on the three chosen cuts $(a, b$, and $c)$ shown in the upper panel.

internal plasma is ejected out of the CS, which leads to the appearance of the turbulent region near the two tips of the CS. In order to evaluate the numerical diffusion responsible for the reconnection, we estimate $\eta_{\text {num }}$, which is $v_{\text {in }} \delta \approx 0.002$ in the red box, where $\delta \approx 0.02$ (i.e. $10^{3} \mathrm{~km}$ ) is the minimum full width of the CS and $v_{\text {in }} \approx 0.1$ is the speed of the reconnection inflow. From this value, we then estimate the Lundquist number $S=L_{\mathrm{c}} v_{\mathrm{c}} / \eta \approx 10^{4}$. Here, $L_{\mathrm{c}} \approx 2 R$ and $v_{\mathrm{c}} \approx 5$ are the characteristic MFR length and the characteristic Alfvén speed near the MFR foot-points. Because particle acceleration is an important aspect related to magnetic reconnection inside the CS, we can also estimate the distribution of the parallel electric field using $E_{\|}=\left(\eta_{\text {num }} \nabla \times \boldsymbol{B}\right) \cdot \hat{\boldsymbol{B}}$ in panel c. It indicates the possible sites where particles of small pitch angle are accelerated to high nonthermal energy (Ripperda et al. 2017).

In Fig. 4, we display the 3D morphology of the CS in a snapshot at $t=9.6$. In order to highlight substructures inside the $\mathrm{CS}$, three planar cuts are chosen, as marked in the upper panel, and current distributions of these cuts are given in the lower panels. The fine structures inside the CS as seen in the planar cuts are very similar to these seen in 2D numerical simulations (Nishida et al. 2009; Shen et al. 2011; Mei et al. 2012; Ni et al. 2015). The magnetic reconnection inside the CS experiences a fractal process because of the tearing mode instabilities (see e.g. Shibata \& Tanuma 2001) and the appearance of bounding slow shocks. When the aspect ratio of the growing CS exceeds 50, 3D tearing mode features start to appear. Here, the aspect ratio of the CS is calculated directly from the 2D studies of tearing instability, which consider the ratio between full width and its vertical (locally slanted) length along the direction of the CS plane.

Different from the 2D case, the 3D tearing mode does not lead to the formation of isolated magnetic islands, although they lead to the appearance of 2D magnetic islands on the cuts shown in Fig. 4. In the third direction, these 2D magnetic islands on the cuts extend in a more random geometry shape on the plane of $\mathrm{CS}$, leading to the appearance of distorted cylindrical flux tube substructures of about $4 \delta$ in width and about 0.9 in length in the 

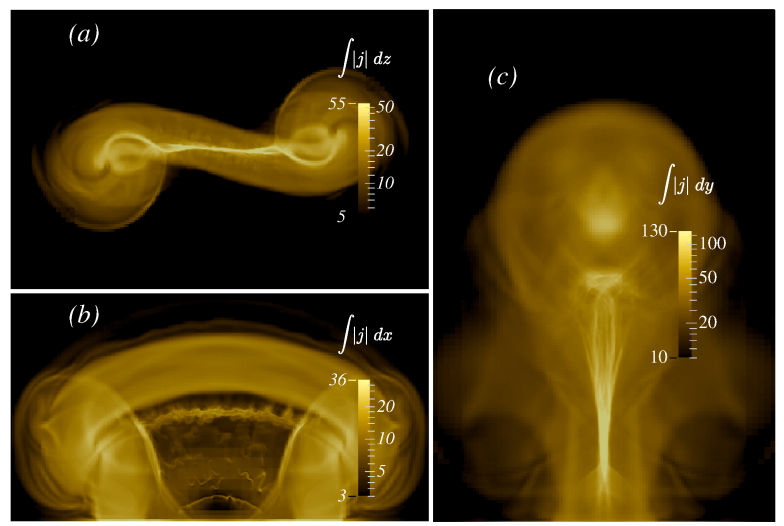

Fig. 5. Evolution snapshots of $\int|j| \mathrm{d} z(a), \int|j| \mathrm{d} x(b)$, and $\int|j| \mathrm{d} y(c)$ at $t=9.6$.

CS plane. These distorted flux tube structures appear as the CS does not experience tearing mode simultaneously in the dimensions normal to and along the CS. Together with the reconnection outflow, these substructures are squeezed out of the CS subsequently, and their lifetimes are $0.6 \sim 1.5$ (i.e. $3.8 \sim 9.7 \mathrm{~min}$ ).

Finally, we present mock synthetic images of the helical distorted MFRs by integration of the current along different angles of view in Fig. 5. As can be seen from these panels, the topological characteristics of MFR highly depend on the viewing angle. Panel a gives the integrated current distribution along the $z$-axis, which demonstrates the appearance of a sigmoid structure during the eruption as a result of the kink instability. Under the sigmoid structure, a bright CS forms and connects two foot-points of MFR. In panel $b$, the integrated current distribution along the $x$-axis shows that the geometrical shape of MFR has been distorted and stretched due to the kink instability and line-tied condition on the photosphere. Below the MFR, we can see a 3D CS structure, the turbulent interaction regions and the distorted-cylindrical substructure in this side view. In panel c, the integrated current distribution along the $y$-axis shows a flareCS-coronal mass ejection (CME) symbiosis, which has a bright core, an underlying flare loop system and a long straight CS in the middle. The bifurcated slow shocks show up clearly in this view. Because of the stronger signal of the long straight $\mathrm{CS}$, the substructure inside the CS does not show up very clearly in this panel. Thus, our synthetic views based on current cannot directly link the distorted cylindrical substructure to the larger blobs observed in the long straight CS by SOHO/LASCO C2.

\section{Conclusion}

We have studied the eruption of a MFR with infinite twist turns on its surface by performing a 3D isothermal MHD numerical experiment. Because of the Kruskal-Shafranov condition, the MFR twists itself immediately after the launch of this simulation. Later, the distortion of MFR implies that the central part of MFR loses its equilibrium and so erupts upward. Because both foot-points of MFR are anchored to the photosphere, the eruptive MFR gets stretched into a long helical structure, and so its geometrical features are highly dependent on the orientation of the line of sight. The eruptive process of MFR manifests as the formation of a sigmoid structure, an outward expansion of distorted MFR, and an eruptive CME followed by a long straight CS when we observe it from the top, the front, and the side, respectively. These three kinds of common phenomena can be interpreted from a single eruption of the MFR.

During the eruption, the rising MFR stretches the magnetic field lines around it, and the field lines and plasma below the
MFR converge toward one another so that a 3D CS forms. Through the CS, the magnetic fields are reconnected and the inflow plasma is accelerated to the local Alfvén speed near the CS. Then these reconnected field lines and accelerated plasma are ejected through the two tips of the CS, hit on the ambient closed magnetic structure above and below the extended 3D CS, and lead to the appearance of two turbulence regions.

With the continuous growth of the CS in its length, the tearing mode instability occurs inside the $\mathrm{CS}$, and there is a clear bifurcating structure which consists of two pairs of slow mode shocks. Because of these internal processes, the reconnection rate is approximated by the Petschek reconnection process, about 4 times larger than the Sweet-Parker reconnection with $S \approx 10^{4}$. In addition, a significant substructure exists in the plane of the CS, which is different from 2D CS. The CS undergoes the tearing mode instability across the CS, but the resultant substructures expand themselves in the other dimension more randomly, which leads to the formation of distorted-cylindrical substructures. These flux tube structures may relate to plasma blobs observed in expanding CS observations.

Acknowledgements. This research has been supported by the Belgian Science Policy Office (www.belspo.be) and the Interuniversity Attraction Poles Programme (initiated by the Belgian Science Policy Office, IAP P7/08 CHARM) and by project GOA/2015-014 (KU Leuven). This work was also supported by the Program 973 grant 2013CBA01503, the NSFC under grants 11303088 and 11573064 , the NSFC-CAS joint grant U1631130, and the CAS grant QYZDJSSW-SH012. Our simulation is conducted on the VSC (Flemish Supercomputer Center) funded by the Hercules foundation and the Flemish government. We acknowledge fruitful discussions with Yang Guo and Chun Xia.

\section{References}

Archontis, V., Hood, A. W., \& Tsinganos, K. 2014, ApJ, 786, L21 Baty, H. 2000, A\&A, 353, 1074

Baty, H., Einaudi, G., Lionello, R., \& Velli, M. 1998, A\&A, 333, 313 Čada, M., \& Torrilhon, M. 2009, J. Comput. Phys., 228, 4118

Forbes, T. G., \& Malherbe, J. M. 1986, ApJ, 302, L67

Forbes, T. G., \& Priest, E. R. 1995, ApJ, 446, 377

Guidoni, S. E., DeVore, C. R., Karpen, J. T., \& Lynch, B. J. 2016, ApJ, 820, 60

Guo, Y., Ding, M. D., Cheng, X., Zhao, J., \& Pariat, E. 2013, ApJ, 779, 157

Janvier, M., Aulanier, G., \& Démoulin, P. 2015, Sol. Phys., 290, 3425

Keppens, R., Meliani, Z., van Marle, A. J., et al. 2012, J. Comput. Phys., 231,

718

Kliem, B., \& Török, T. 2006, Phys. Rev. Lett., 96, 255002

Kliem, B., Su, Y. N., van Ballegooijen, A. A., \& DeLuca, E. E. 2013, ApJ, 779, 129

Kliem, B., Lin, J., Forbes, T. G., Priest, E. R., \& Török, T. 2014, ApJ, 789, 46

Leake, J. E., Linton, M. G., \& Török, T. 2013, ApJ, 778, 99

Li, L., \& Zhang, J. 2009, ApJ, 703, 877

Lin, J., \& Forbes, T. G. 2000, J. Geophys. Res., 105, 2375

Lin, J., van Ballegooijen, A. A., \& Forbes, T. G. 2002, J. Geophys. Res. (Space Physics), 107, 1438

Lin, J., Ko, Y.-K., Sui, L., et al. 2005, ApJ, 622, 1251

Masuda, S., Kosugi, T., Hara, H., Tsuneta, S., \& Ogawara, Y. 1994, Nature, 371, 495

Mei, Z., Shen, C., Wu, N., et al. 2012, MNRAS, 425, 2824

Mei, Z. X., Keppens, R., Lin, J., \& Roussev, I. I. 2017, A\&A, submitted

Ni, L., Kliem, B., Lin, J., \& Wu, N. 2015, ApJ, 799, 79

Nishida, K., Shimizu, M., Shiota, D., et al. 2009, ApJ, 690, 748

Nishida, K., Nishizuka, N., \& Shibata, K. 2013, ApJ, 775, L39

Porth, O., Xia, C., Hendrix, T., Moschou, S. P., \& Keppens, R. 2014, ApJS, 214,

4

Ripperda, B., Porth, O., Xia, C., \& Keppens, R. 2017, MNRAS, 467, 3279

Roussev, I. I., Forbes, T. G., Gombosi, T. I., et al. 2003, ApJ, 588, L45

Schanche, N. E., Reeves, K. K., \& Webb, D. F. 2016, ApJ, 831, 47

Shen, C., Lin, J., \& Murphy, N. A. 2011, ApJ, 737, 14

Shibata, K., \& Tanuma, S. 2001, Earth Planets Space, 53, 473

Su, Y., Veronig, A. M., Holman, G. D., et al. 2013, Nature Phys., 9, 489

Takasao, S., Asai, A., Isobe, H., \& Shibata, K. 2012, ApJ, 745, L6

Titov, V. S., \& Démoulin, P. 1999, A\&A, 351, 707

Török, T., Kliem, B., \& Titov, V. S. 2004, A\&A, 413, L27

Vasyliunas, V. M. 1975, Rev. Geophys. Space Phys., 13, 303 